Check for updates

Cite this: Mater. Chem. Front., 2019, 3, 1105

Received 26th February 2019, Accepted 29th March 2019

DOI: $10.1039 / \mathrm{c} 9 q m 00124 g$

rsc.li/frontiers-materials

\section{A stabilized lamellar liquid crystalline phase with aggregation-induced emission features based on pyrrolopyrrole derivatives $\dagger$}

\author{
Shuangxiong Dai, ${ }^{a}$ Zhengxu Cai, (D) ${ }^{a}$ Zhe Peng, ${ }^{a}$ Zhi Wang, ${ }^{a}$ Bin Tong, (D) *a \\ Jianbing Shi, (D) ${ }^{a}$ Shenglong Gan, ${ }^{\mathrm{b}}$ Qiming He, Wei Chen (D) ${ }^{\mathrm{bc}}$ and \\ Yuping Dong (D) *a
}

\begin{abstract}
Aggregation-induced emission (AIE) overcoming the drawback of aggregation-caused quenching (ACQ) makes it possible to access the fluorescence emission of chromophores in the solid state. The development of AIE-active liquid crystals has attracted extensive attention in recent years. Herein, a series of novel (A-D-A)-type AlE-active dyes with different lengths of flexible alkyl chains based on pyrrolopyrrole cores were prepared. Among these compounds, TPPP-C1,2,3 with shorter alkyl chains possessed a more excellent AIE performance; however, they were unable to form a liquid crystalline (LC) phase. Upon increasing the length of alkyl chains, TPPP-C4,5,6,7,8,12 exhibited declining fluorescence emission efficiency in the aggregation states, and only TPPP-C6,7,8,12 with even longer alkyl chains exhibited the LC properties. Thus, all the nine TPPP compounds were AIE-active dyes, whereas TPPP-C6,7,8,12 were AIE-active liquid crystals. Polarized optical microscopy and differential scanning calorimetry experimental results indicated that the LC phases of the four AIE-active liquid crystals were only formed during the cooling process. However, once formed, the LC phases were very stable and could be retained for several months. This might be due to the freezing of intertwined alkyl chains during the cooling process; this was supported by grazing incidence wide-angle $\mathrm{X}$-ray scattering measurements. The models of molecular organization in the LC states were built, and they showed well-organized lamellar structures.
\end{abstract}

\section{Introduction}

The development and utilization of liquid crystals (LCs) has made significant advances. ${ }^{1,2}$ In recent years, luminescent display materials, such as light-emitting liquid crystalline (LC) displays,${ }^{3-9}$ organic light-emitting diodes,${ }^{10,11}$ organic optoelectronics, ${ }^{12,13}$ and sensors, ${ }^{14-17}$ have attracted researchers' interests due to their growing applications and requirements. However, many luminescent molecules are highly emissive in

\footnotetext{
${ }^{a}$ Beijing Key Laboratory of Construction Tailorable Advanced Functional Materials and Green Applications, School of Material Science \& Engineering, Beijing Institute of Technology, 5 South Zhongguancun Street, Beijing, 100081, China. E-mail: tongbin@bit.edu.cn, chdongyp@bit.edu.cn

${ }^{b}$ Materials Science Division, Argonne National Laboratory, 9700 Cass Avenue, Lemont, Illinois 60439, USA

${ }^{c}$ Institute for Molecular Engineering, The University of Chicago,

5640 South Ellis Avenue, Chicago, Illinois 60637, USA

$\dagger$ Electronic supplementary information (ESI) available: Details of synthesis and characterization, UV-vis absorption and PL emission spectra, time-resolved PL decays spectra, TGA, XRD, POM, DSC, and single crystal data. CCDC 1882581 (TPPP-C6). For ESI and crystallographic data in CIF or other electronic format see DOI: $10.1039 / \mathrm{c} 9 \mathrm{qm} 00124 \mathrm{~g}$
}

solution but quenched in aggregate states. This phenomenon has been documented as aggregation-caused quenching (ACQ). ${ }^{18}$ There remain challenges to obtain high-efficiency luminous materials.

In 2001, Tang and co-workers reported a novel phenomenon of the aggregation-induced emission (AIE) of some propellershaped molecules. ${ }^{18-20}$ Owing to the twisted configuration, AIE molecules show opposite features with the ACQ phenomenon: highly emissive in the aggregate states but quenched in pure solutions. This twisted structure greatly restricts the strong intermolecular $\pi-\pi$ interactions; thus, the emission efficiency is significantly increased..$^{21-24}$ The utilization of the AIE phenomenon to synthesize luminescent LCs is attracting extensive attention from researchers.

To date, researchers have synthesized some luminescent LCs. Wang and Lai et al. reported the preparation of AIE-LCs based on 2,3,4,5-tetraphenylsilole functionalized with long-chain alkoxydiacylamido. ${ }^{25}$ They used silole as the AIE core and benzene-amide-benzene as LC units. The two derivatives were self-assembled into a LC phase over a wide temperature range, and these were affected by the length of alkyl chains. Tang et al. 

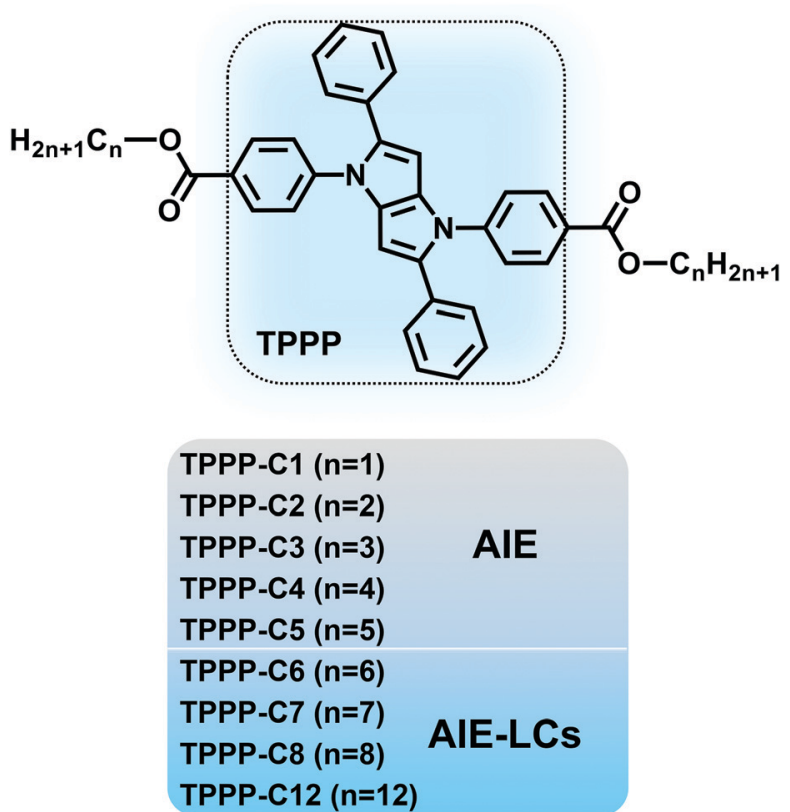

Scheme 1 Molecular structures of the nine TPPP derivatives. TPPPC1,2,3,4,5 with AIE properties and TPPP-C6,7,8,12 with both AIE and LC properties.

described the preparation of TPE4Mes, ${ }^{26}$ which took tetraphenylethylene (TPE) as the AIE core linked with four peripheral mesogenic substituents. TPE4Mes combined both AIE and LC features. Two different smectic phases, respectively, emerged at $194.2{ }^{\circ} \mathrm{C}$ and $140.8{ }^{\circ} \mathrm{C}$ upon cooling from the isotropic state. Wang et al. prepared two kinds of AIE-LCs, ${ }^{10}$ which took TPE as the emissive core and a 4-cynobiphenyl moiety as the mesogenic unit. The LC phases of the two AIE-LCs were observed in the heating process, and they were self-assembled into smectic and oblique phases, respectively. The published AIE-LC research works mainly focused on employing TPE as AIE-active cores. ${ }^{5,6,9,11,27-31}$ Fewer other kinds of AIE units have been adopted to synthesize AIE-LC compounds. ${ }^{14,32-35}$

In this paper, we constructed AIE-LCs based on the AIEgens of 1,2,4,5-tetraphenyl-1,4-dihydropyrrolo[3,2-b]-pyrrole (TPPP) developed by our groups. ${ }^{36,37}$ As shown in Scheme 1, a series of TPPP derivatives (TPPP-Cn) were synthesized. TPPP with typical propeller-like structures served as the AIE activity units, then different lengths of flexible chains were linked to this TPPP core through ester groups on benzene rings in the 1,4-position. The research results showed that all the compounds (TPPP-C1$\mathbf{8 , 1 2}$ ) were AIE-active dyes, while TPPP-C6,7,8,12 also exhibited LC properties and were proved to be AIE-active LCs. TPPP was first reported as a functional unit exhibiting both AIE and LC properties, which could lead to the development of new functional materials.

\section{Experimental}

\subsection{Synthesis of TPPP derivatives}

The synthetic routes for the TPPP derivatives are shown in Scheme $\mathrm{S} 1$ in the ESI, $\uparrow$ and the preparation method is available as reported in the literature. ${ }^{38,39}$ Despite the low yields (10-14\%), these TPPP derivatives could be easily obtained using only one synthetic step. All the TPPP derivatives were characterized by ${ }^{1} \mathrm{H}$ NMR and ${ }^{13} \mathrm{C}$ NMR spectroscopy and MALDI-MS to confirm their structures.

\subsection{Grazing incidence wide-angle X-ray scattering measurements}

Grazing incidence wide-angle X-ray scattering (GIWAXS) measurements were performed using Beamline 8ID-E at the Advanced Photon Source (APS), Argonne National Laboratory, using X-rays with a wavelength of $\lambda=1.136 \AA$ and a beam size of $\sim 200 \mu \mathrm{m}$ (horizontal) and $20 \mu \mathrm{m}$ (vertical). The molecular organization of TPPP-C6 and TPPP-C12 in the LC phase were investigated by GIWAXS. The samples of LC phase thin film were obtained by cooling from the isotropic liquid phase at a very slow rate of $0.5{ }^{\circ} \mathrm{C} \mathrm{min}{ }^{-1}$ on $\mathrm{Si}$ substrates. A twodimensional (2D) area detector was used to capture the scattering patterns from the prepared samples for the GIWAXS measurements. $q_{y}$ and $q_{z}$ scans are the horizontal and vertical linecuts of the 2D GIWAXS patterns, which represent the information related to the parallel and perpendicular wave vector transfers with respect to the substrate surface, respectively. The scattering vector $q$ is expressed by $4 \pi \sin \theta / \lambda$ and the $d$-spacing of a peak is expressed by $2 \pi / q(q=4 \pi \sin \theta / \lambda, d=2 \pi / q)$, where $\theta$ is the half scattering angle.

\subsection{Materials and measurement}

Aniline derivatives were commercially obtained from Energy Chemical Company Ltd and J\&K Chemical Company. Other chemicals were purchased from Aladdin and Beijing Chemical Reagent companies. ${ }^{1} \mathrm{H}$ NMR and ${ }^{13} \mathrm{C}$ NMR spectra were measured on a Bruker AV 400 instrument using $\mathrm{CDCl}_{3}$ as a solvent. Mass spectra (MS) were collected using a Bruker Autoflex III MALDI-TOF instrument. UV-vis spectra were recorded on a TU-1901 double beam UV-vis spectrophotometer. Fluorescence spectra were measured on a Hitachi F-7000 fluorescence spectrophotometer. The absolute fluorescence quantum yield and fluorescence lifetime measurements were acquired using an Edinburgh FLS980 lifetime and steady state spectrometer. The temperature-dependent fluorescence spectra were also recorded on the Edinburgh FLS980 lifetime and steady state spectrometer using an Oxford Optistat DN2 heating unit, and the temperature was controlled by ITC 503 Version 4.01 (c) software. Thermogravimetric analysis (TGA) was carried out on a Shimadzu DTG-60 at a heating rate of $10{ }^{\circ} \mathrm{C} \mathrm{min}-1$ under a nitrogen flow. A differential scanning calorimeter (DSC) was measured on a TA DSC Q2000. Polarized optical microscopy (POM) was carried out using a Nikon LV100N Polar Microscope equipped with a heating stage. Density functional theory (DFT) calculations were performed using the Gaussian 09 package. The crystallographic structures were analyzed on a Rigaku CCD Saturn 724+ X-ray single crystal diffractometer with an X-ray beam wavelength of $0.71073 \mathrm{~nm}$.

CCDC 1882581 (TPPP-C6). $\dagger$ 


\section{Results and discussion}

\subsection{Photophysical properties}

The UV-vis absorption spectra of the TPPP derivatives were measured in THF solution at room temperature (Fig. 1), and the relevant data are shown in Table 1 . The nine TPPP derivatives exhibited almost similar absorption peaks. The higher peaks at $\sim 322 \mathrm{~nm}$ are attributed to $\pi-\pi^{*}$ transition of the conjugated molecules, and the weaker peaks at $\sim 274 \mathrm{~nm}$ ascribed to $\pi-\pi^{*}$ transition of the benzene rings. The additional weaker shoulder peaks at $\sim 370 \mathrm{~nm}$ represent intramolecular charge transfer (ICT) absorption between the ester groups and pyrrolo[3,2- $b]$ pyrrole core. These nine TPPP derivatives are acceptor-donoracceptor (A-D-A)-type compounds with a pyrrolo[3,2-b]pyrrole core as the donor, ${ }^{39}$ and ester groups on both sides as the acceptors. So they showed typical ICT absorption peaks.

The emission spectra of the TPPP derivatives in THF also showed similar emission peaks at $\sim 510 \mathrm{~nm}$, which indicate the length of alkyl chain has no significant impact on either absorption or emission spectra. Additionally, all these compounds had large Stokes shift over $130 \mathrm{~nm}$ (Table 1), which means these TPPP derivatives had little self-quenching effect between the emission and excitation. ${ }^{40}$ Therefore, these compounds have potential application in biochemistry, such as fluorescent bio-probes and in cell imaging. ${ }^{41}$ Their UV-vis absorption and PL emission spectra in the solid states are presented in Fig. S1 (ESI $\dagger$ ).

The AIE characteristics were investigated in $\mathrm{THF} / \mathrm{H}_{2} \mathrm{O}$ mixtures since water is a poor solvent for the TPPP derivatives. TPPP-C6 is taken as an example to discuss, and its fluorescence spectra in $\mathrm{THF} / \mathrm{H}_{2} \mathrm{O}$ mixtures with different water fractions are shown in Fig. 2a. In pure THF solution, a weak emission peak at $508 \mathrm{~nm}$ was observed. After the addition of water into the THF solution, the emission intensities decreased and were further red-shifted with the water fraction $\left(f_{\mathrm{w}}\right)$ increasing from $10 \%$ to $40 \%$ (Fig. $2 \mathrm{~b}$ ). This decreased emission intensity and a red-shifted emission wavelength with an increase in the polarity of the solvent, which is

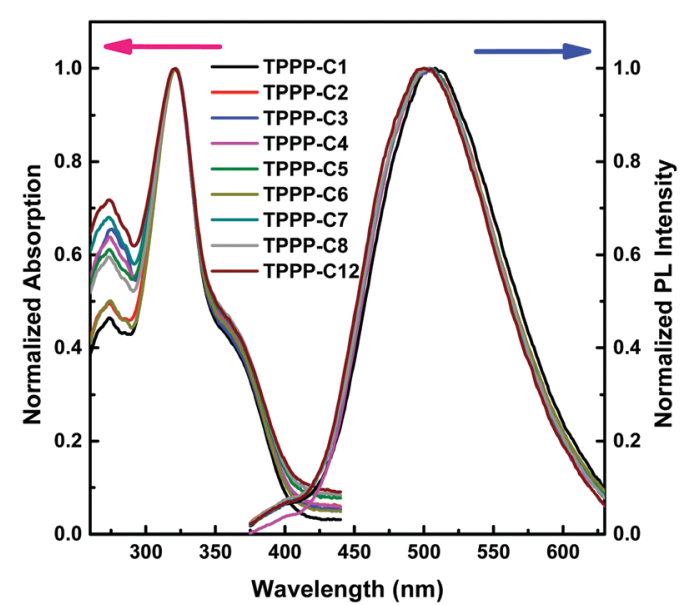

Fig. 1 Normalized UV-vis absorption (left) and fluorescence (right) spectra of TPPP derivatives in THF solution. Concentration: $1 \times 10^{-5} \mathrm{M}$, excitation wavelength: $322 \mathrm{~nm}$. known as intramolecular charge transfer (ICT), are typical photophysical phenomena for D-A type fluorophores. ${ }^{42-44}$ When $f_{\mathrm{w}}$ increased from $50 \%$ to $99 \%$, a blue-shift in emission was observed and the emission intensity was gradually intensified. Finally, a 7.0-fold enhancement was obtained with $f_{\mathrm{w}}=99 \%$. As shown in Fig. 3 and Fig. S3 (ESI $\dagger$ ), other TPPP compounds had the same emission characteristics in $\mathrm{THF} / \mathrm{H}_{2} \mathrm{O}$ mixtures. Furthermore, the fluorescence quantum yield (FQY) of TPPP-C6 in THF and solid powder was $1.27 \%$ and $16.56 \%$, respectively. These results indicated that TPPP-C6 as well as the other TPPP derivatives possessed AIE characteristics.

In order to investigate the relationship between AIE behaviors and the length of peripheral alkyl chains, the enhancement of PL intensity in the THF/water mixtures (Fig. 3) and the FQY (Table 1) of TPPP derivatives are discussed. Increasing the length of peripheral alkyl chains, the FQY exhibited a rising tendency in THF solutions and a declining trend in the solid states. The $I / I_{0}$ values of the nine compounds also showed a decreasing tendency with a $99 \%$ water fraction. These results imply that the nine TPPP derivatives with shorter alkyl chains possess better AIE performance. Thus, increasing the length of alkyl chains is unfavorable to enhance the emission efficiency in aggregation states.

\subsection{DSC and POM measurements}

Thermal treatment is the main method to realize the mesophase of thermotropic liquid crystals. The mesophase features of the nine compounds were checked by TGA, DSC, POM, and GIWAXS. Results showed that TPPP-C1,2,3,4,5 with shorter alkyl chains were not LC compounds, while TPPP-C6,7,8,12 with longer alkyl chains exhibited LC phase features. Therefore, TPPP-C6,7,8,12 were AIE-active liquid crystals.

The DSC measurements were performed during the first cooling and second heating processes with a rate of $5{ }^{\circ} \mathrm{C} \mathrm{min}^{-1}$. For TPPP-C6 (Fig. 4a), there was only one exothermic peak at $107.7{ }^{\circ} \mathrm{C}$ upon cooling from the isotropic liquid phase, and a single transition peak at $158{ }^{\circ} \mathrm{C}$ from the second heating process. TPPP-C12 (Fig. 4b) and TPPP-C7,8 (Fig. S8, ESI $\dagger$ ) showed similar DSC thermograms, where only one transition peak was found both from the first cooling and second heating process.

The LC phase textures of TPPP-C6,12 (Fig. 4c and d) and TPPP-C7,8 (Fig. S7, ESI $\dagger$ ) investigated by POM were observed only upon the cooling process occurring at a very slow rate of $0.5{ }^{\circ} \mathrm{C} \mathrm{min}^{-1}$ from the isotropic liquid phase, indicating that they were monotropic LCs. The POM images of TPPP-C6,7,8 revealed focal-conic fan-shaped textures, while TPPP-C12 showed banded textures. These belong to smectic textures, which was further confirmed by GIWAXS. We also noted that the crystalline phase of TPPP-C6,12 (Fig. 4e and f) were observable when the cooling rate was over $5{ }^{\circ} \mathrm{C} \min ^{-1}$. Therefore, a slow cooling rate led to the formation of LC phase, while rapid cooling generated the crystalline phase.

This phenomenon also occurred for the normal LC compounds. Hegmann et al. reported a bent-core liquid crystal, ${ }^{45}$ and that different LC phases were obtained by cooling slowly from the 
Table 1 Photophysical properties of the nine TPPP derivatives in solution and in the solid state

\begin{tabular}{|c|c|c|c|c|c|c|c|c|c|c|c|}
\hline Comps & $\begin{array}{l}\text { Abs/THF } \\
(\mathrm{nm})\end{array}$ & $\begin{array}{l}\mathrm{Em} / \mathrm{THF} \\
(\mathrm{nm})\end{array}$ & $\begin{array}{l}\text { Stoke's shift } \\
\text { (nm) }\end{array}$ & $\begin{array}{l}\text { Em/solid } \\
\text { (nm) }\end{array}$ & $\begin{array}{l}\Phi_{\mathrm{F}} / \mathrm{THF} \\
(\%)\end{array}$ & $\begin{array}{l}\Phi_{\mathrm{F}} / \text { solid } \\
(\%)\end{array}$ & $\begin{array}{l}\tau / \mathrm{THF} \\
\text { (ns) }\end{array}$ & $\begin{array}{l}\tau / \text { solid } \\
\text { (ns) }\end{array}$ & $\begin{array}{l}\text { HOMO } \\
(\mathrm{eV})\end{array}$ & $\begin{array}{l}\text { LUMO } \\
(\mathrm{eV})\end{array}$ & $\begin{array}{l}E_{\mathrm{g}} \\
(\mathrm{eV})\end{array}$ \\
\hline TPPP-C1 & $274,321,372$ & 505 & 133 & 476 & 1.11 & 29.37 & 2.95 & 15.4 & -5.18 & -1.80 & 3.38 \\
\hline TPPP-C2 & $273,321,368$ & 510 & 142 & 465 & 1.27 & 24.28 & 3.09 & 8.86 & -5.16 & -1.76 & 3.40 \\
\hline TPPP-C4 & $274,321,374$ & 512 & 138 & 461 & 1.24 & 14.81 & 3.24 & 15.39 & -5.15 & -1.74 & 3.41 \\
\hline TPPP-C5 & $274,321,367$ & 512 & 145 & 462 & 1.38 & 13.87 & 3.28 & 14.09 & -5.15 & -1.74 & 3.41 \\
\hline TPPP-C6 & $275,321,371$ & 507 & 136 & 466 & 1.27 & 16.56 & 3.27 & 9.29 & -5.15 & -1.75 & 3.40 \\
\hline TPPP-C12 & $274,321,372$ & 506 & 134 & 468 & 1.48 & 14.25 & 3.30 & 8.37 & -5.15 & -1.75 & 3.40 \\
\hline
\end{tabular}
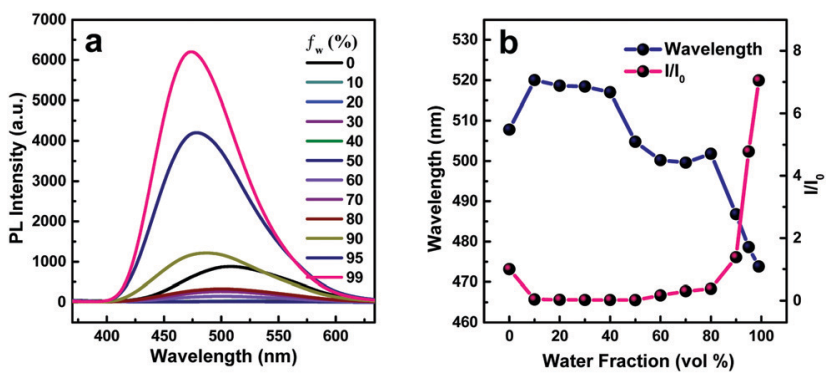

Fig. 2 (a) Fluorescence spectra of TPPP-C6 in THF/water mixtures with different water fractions $\left(f_{\mathrm{w}}: 0-99 \%\right)$; (b) plot of the wavelength and the ratio of the maximum fluorescence intensity of TPPP-C6 vs. the water fraction, $I_{0}=$ emission intensity in pure THF solution. Excitation wavelength: $322 \mathrm{~nm}$, concentration: $1 \times 10^{-5} \mathrm{M}$.

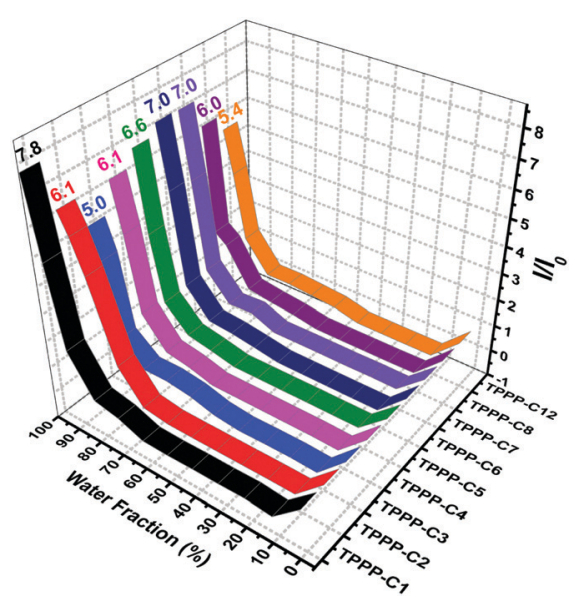

Fig. 3 Plot of the ratio of the maximum fluorescence intensity $\left(I / I_{0}\right)$ of the nine TPPP derivatives vs. the water fraction.

isotropic liquid state (at rates of $0.5,2$, or $5{ }^{\circ} \mathrm{C} \min ^{-1}$ ). In addition, Alaasar et al. described a type of azobenzene liquid crystal. ${ }^{46}$ Upon cooling from the isotropic liquid phase with a low rate of $\leq 2{ }^{\circ} \mathrm{C} \mathrm{min}^{-1}$, the LC phase was formed. However, a crystalline phase occurred upon cooling with a faster rate of $\geq 5{ }^{\circ} \mathrm{C} \min ^{-1}$.

Once the LC phases (TPPP-C6,7,8,12) were formed, they were stable and no other phase transformation were observed during the further cooling to room temperature. The kinds of LC phases could be immobilized and made stable to be retained
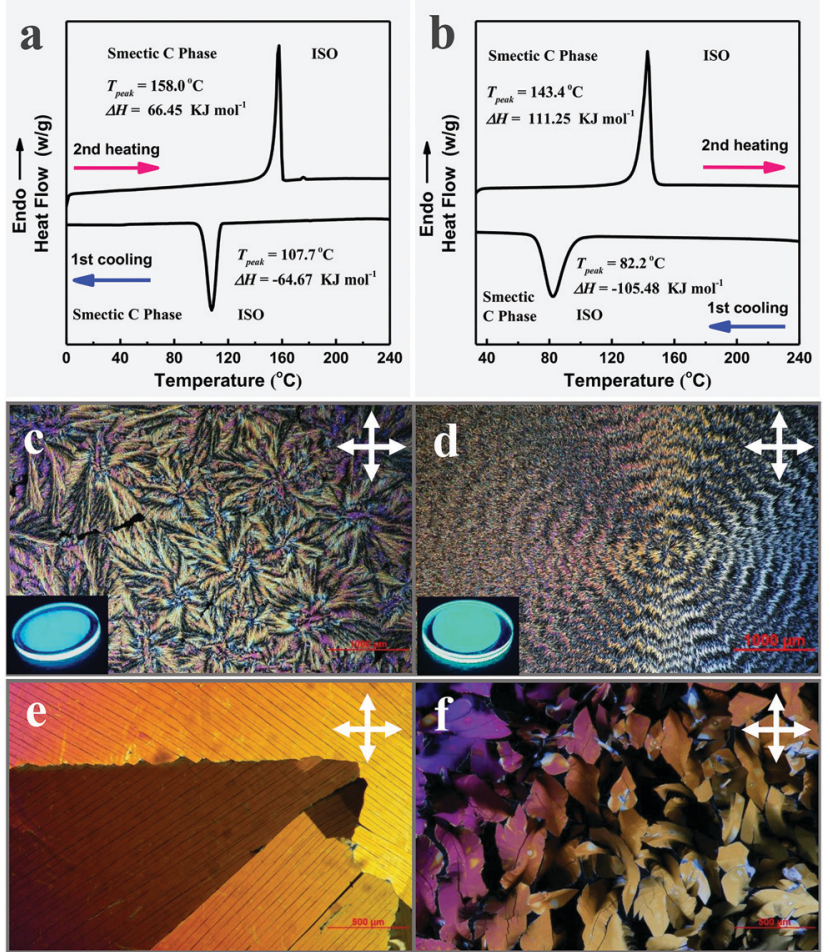

Fig. 4 DSC curves of TPPP-C6 (a) and TPPP-C12 (b) recorded under nitrogen during the first cooling and second heating cycles with a scan rate of $5{ }^{\circ} \mathrm{C} \mathrm{min}^{-1}$. Mesomorphic textures observed on cooling TPPP-C6 (c) to $103{ }^{\circ} \mathrm{C}$ and TPPP-C12 (d) to $87^{\circ} \mathrm{C}$ at a cooling rate of $0.5^{\circ} \mathrm{C} \mathrm{min}{ }^{-1}$, the inset emission photos of the LC phase taken under UV light. POM patterns of TPPP-C6 (e) and TPPP-C12 (f) in the crystalline phase with a faster cooling rate of over $5{ }^{\circ} \mathrm{C} \mathrm{min}^{-1}$.

at room temperature for several months. Because of there being no additional phase transformations during the further cooling process, the DSC curves showed only one transition peak.

\subsection{GIWAXS measurements}

To characterize the structures of the LC phase, we performed 2D GIWAXS measurements using a synchrotron X-ray radiation source and built models of the molecular organization in the LC phase (Fig. 5). In the low- $q$ region, TPPP-C6 exhibited distinct peaks in the direction of $q_{y}=0.30,0.60,0.91 \AA^{-1}$ (Fig. 5b) and $q_{z}=0.31,0.62,0.91 \AA^{-1}$ (Fig. 5c), which arose from the (100), (200), (300) Bragg diffraction peaks. The scattering 


\section{a}

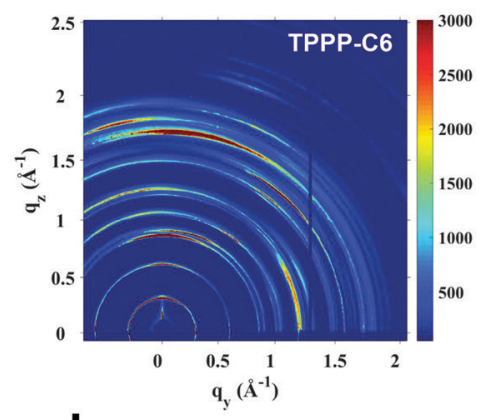

d
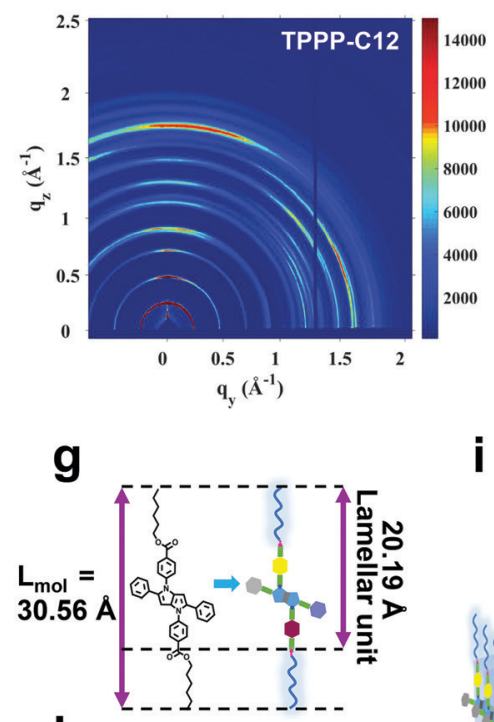

h

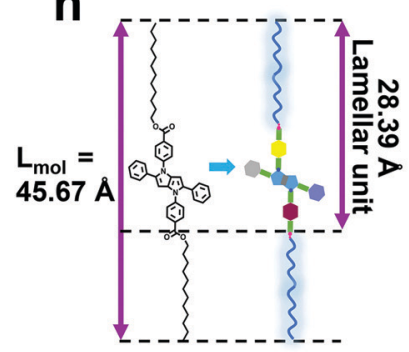

i b

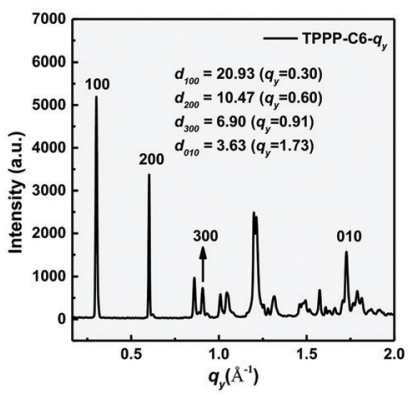

e
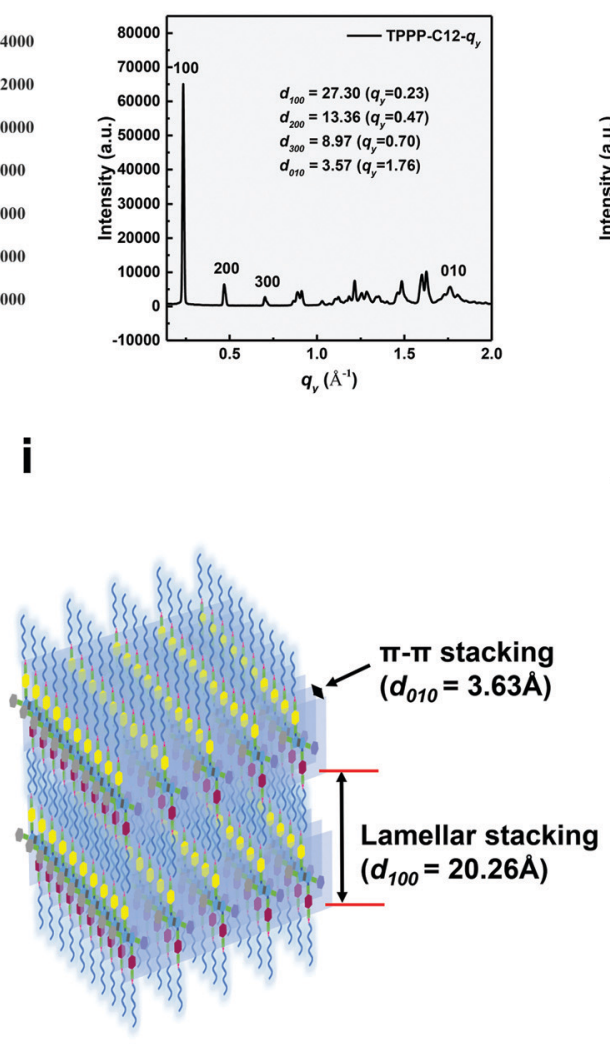

\section{C}
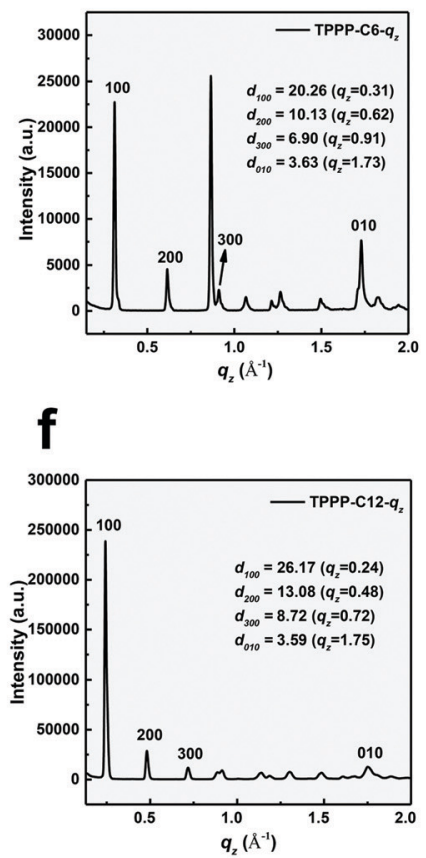

j

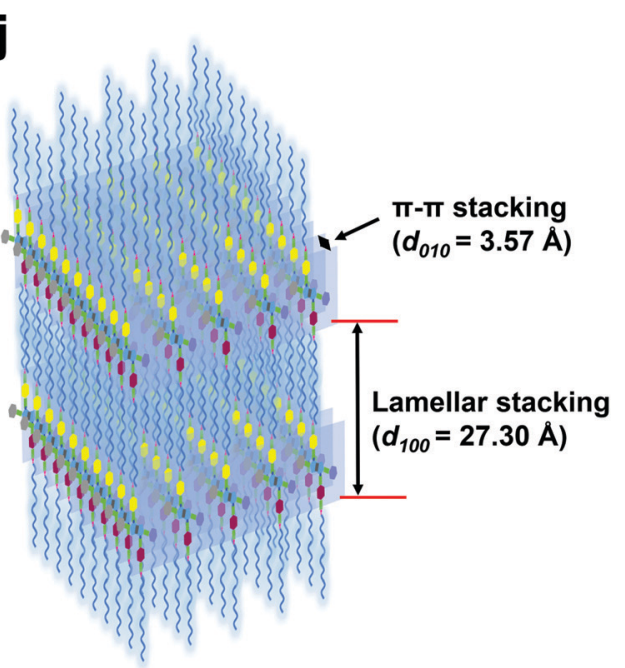

Fig. 5 2D GIWAXS patterns ( $a$ and d), horizontal line-cuts ( $b$ and e) and vertical line-cuts ( $c$ and $f$ ) of TPPP-C6 and TPPP-C12 LC films measured at room temperature, respectively; molecular size of TPPP-C6 (g) and TPPP-C12 (h); models of the molecular organization in the LC phase of TPPP-C6 (i) and TPPP-C12 (j).

peaks in low- $q$ region indicated well-organized lamellar structures in the LC states. In the high- $q$ region, several diffractions were partially overlapped, especially in the $q_{y}$ direction, which can be identified with the ordered arrangement of the peripheral alkyl chains (Fig. 5b and c). In addition, a diffraction peak appeared at $1.73 \AA^{-1}$, both in the $q_{y}$ and $q_{z}$ direction, and can be ascribed to the (010) peak, which is associated with the intermolecular $\pi-\pi$ stacking distance $\left(d_{010}=3.63 \AA, d=2 \pi / q\right)$. It is noteworthy that the diffraction peaks in the $q_{z}$ direction were much stronger than in the $q_{y}$ direction, which suggests that the molecules stacking was primarily perpendicular to the substrate and the $\pi$-faces were approximately parallel to the substrate. ${ }^{47-50}$
In order to build a model for the molecular organization in the LC phase of TPPP-C6, the molecular size was measured using the Gaussian 09 software. As shown in Fig. 5g, the molecular length of TPPP-C6 was evaluated to be $30.56 \AA$. Moreover, the $d$-spacing of the primary diffraction peaks $\left(d_{100}\right)$ were $20.93 \AA$ in the $q_{y}$ direction and $20.26 \AA$ in the $q_{z}$ direction, which are close to the lamellar unit (20.19 ̊) we calculated in Fig. 5g. So we can conclude that when TPPP-C6 self-assembled into a layer structure in the LC state, the peripheral alkyl chains were intertwined with each other and the molecules of TPPP-C6 adopted an overlapped head-to-tail configuration. According to the distance of lamellar stacking $\left(d_{100}\right)$ 
and $\pi-\pi$ stacking $\left(d_{010}\right)$, a model of TPPP-C6 in the stabilized LC phase was built and is shown in Fig. 5i. The LC phase structures of TPPP-C12 were also characterized by GIWAXS. As shown in Fig. 5e and f, TPPP-C12 exhibited distinct peaks in the direction of $q_{y}=$ $0.23,0.47,0.70 \AA^{-1}$ and $q_{z}=0.24,0.48,0.72 \AA^{-1}$ in the low- $q$ region, which could be indexed as the (100), (200), (300) diffraction peaks. In the high- $q$ region, the partially overlapped peaks could be identified with the peripheral alkyl chains. The diffraction peaks at $1.76 \AA^{-1}$ in the $q_{y}$ direction and $1.75 \AA^{-1}$ in the $q_{z}$ direction could be ascribed to the (010) peaks, which are associated with the $\pi-\pi$ stacking distance $\left(q_{y}: d_{010}=3.57 \AA ; q_{z}: d_{010}=3.59 \AA\right)$. Similar to the TPPP-C6, TPPP-C12 also exhibited a well-organized lamellar structure in the LC states (Fig. 5j). With the increasing alkyl chain length, the distances $\left(d_{100}\right)$ between two layers increased. Additionally, the value of $d_{100}$ is smaller than the single molecular length $\left(L_{\mathrm{mol}}\right)$ and obviously larger than $L_{\text {mol }} / 2$, indicating a tilted layer structure in the LC phases. The ratio of $d_{100} / L_{\mathrm{mol}}$ was $\sim 0.68$ for TPPP-C6 and 0.60 for TPPP-C12. According to $d / L_{\mathrm{mol}}=\cos \beta$, the tilt angle was around $47^{\circ}$ and $53^{\circ}$ for the two compounds. So the LC states of TPPP-C6 and TPPP-C12 are assigned as smectic C phase.

\subsection{Temperature-dependent fluorescence measurements}

To investigate the photophysical properties in the LC state, we performed temperature-dependent fluorescence spectroscopy of TPPP-C6 during the temperature decreasing from $180{ }^{\circ} \mathrm{C}$ to $20{ }^{\circ} \mathrm{C}$ (Fig. 6a). Fig. 6b shows the changes in the maximum wavelengths and relative $\mathrm{PL}$ intensities during the cooling process. The first region is associated with liquid states in the higher temperature range $\left(180-120{ }^{\circ} \mathrm{C}\right)$, where both the maximum wavelengths and the PL intensities had no obvious change with the temperature decrease. The weak emission intensity in the first region was caused by a non-radiative decay process and the relaxation of excited energy was primarily via the thermal motion of the molecules. The second region is the LC-state (120-100 ${ }^{\circ} \mathrm{C}$ ), where an evident blue-shift of PL maximum wavelength with the increasing PL intensity was observed with the formation of highly ordered structures in the LC state leading to AIE properties. In the third region, the LC state was formed. The maximum wavelength exhibited a tendency to red-shift, but the changes were minor. The PL
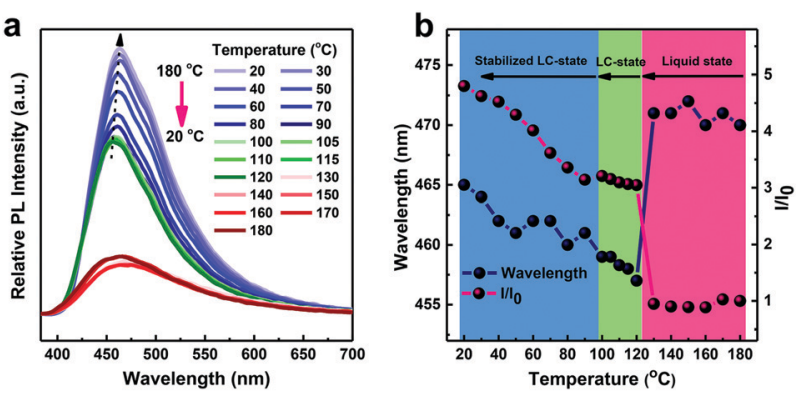

Fig. 6 Temperature-dependent fluorescence spectra (a) and plot of wavelength and fluorescence intensity vs. temperature of TPPP-C6 (b) during the cooling process from $180{ }^{\circ} \mathrm{C}$ to room temperature under $360 \mathrm{~nm}$ UV light. intensity continuously increased with the temperature decreasing to $20{ }^{\circ} \mathrm{C}$, originating from the further restriction of molecular motions and the enhancement of the molecular radiative channels at low temperature.

\subsection{Crystal structure analysis}

The accurate structure of a single crystal helps us to understand the relationship of the molecular stacking style with the relevant photophysical properties. The single crystal of TPPP-C6 was obtained and characterized by X-ray single crystal diffractometry. Its crystal structure was a triclinic crystal system and the relevant crystal data are summarized in Table S1 (ESI $\dagger$ ). The dihedral angles between the central pyrrolo[3,2-b]pyrrole core and its substitutes of benzene were $52.25^{\circ}$ and $41.91^{\circ}$, respectively (Fig. 7a). In addition, the longer distances (4.62-6.33 $\mathrm{A})$ between the benzene rings and pyrrolo[3,2- $b]$ pyrrole cores manifested the lack of intermolecular $\pi-\pi$ stacking interactions (Fig. 7b). On the other hand, several kinds of inter- and intramolecular $\mathrm{C}-\mathrm{H} \cdots \pi$ as well as $\mathrm{C}-\mathrm{H} \cdots \mathrm{O}$ interactions existed between the adjacent TPPP-C6 molecules, as shown in Fig. 7c. Such kinds of non-covalent interactions work together to help the formation of stabilized crystal packing, and restrict the rotational motions of the phenyl rings. Therefore, the non-radiative channel is restrained, while the radiative pathway is opened, leading to TPPP-C6 being an AIE molecule. The peripheral alkyl chains overlapped with each other (Fig. 7d), which is similar to the models we suggested in the LC phase.

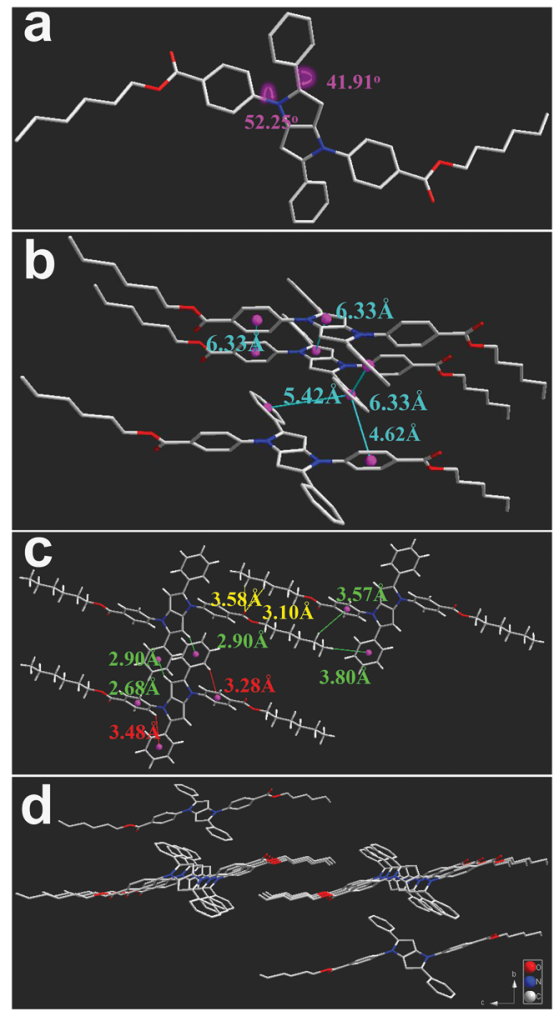

Fig. 7 The crystal structure (a), intermolecular interactions (b and c), and packing patterns (d) of TPPP-C6. Green and red lines represent the interand intramolecular $\mathrm{C}-\mathrm{H} \cdots \pi$ interactions, respectively, and yellow lines represent the $\mathrm{C}-\mathrm{H} \ldots \mathrm{O}$ interactions in Fig. 8(c). 


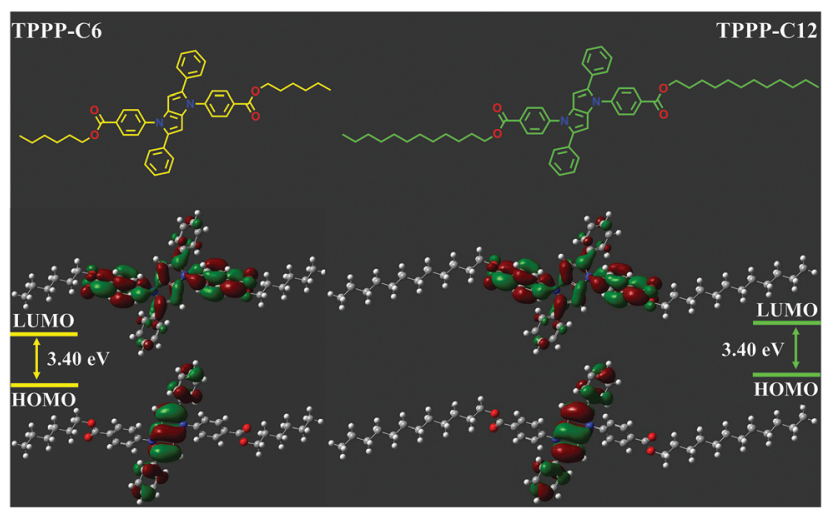

Fig. 8 Calculated electron distributions of HOMOs-LUMOs and the energy levels of TPPP-C6 (left) and TPPP-C12 (right).

\subsection{Theory calculations}

To further investigate the relationship between the molecular structure and photophysical properties of the TPPP derivatives, density functional theory (DFT) calculations were performed using the Gaussian 09 program. The highest occupied molecular orbitals (HOMOs) and the lowest unoccupied molecular orbitals (LUMOs) of TPPP-C6 and TPPP-C12 were calculated at the B3LYP/ 6-31G(d) level after structural optimization (Fig. 8). The two compounds had similar HOMOs and LUMOs, indicating that the alkyl chains had no effects on the distributions of electrons. That is why TPPP-C6 and TPPP-C12 exhibited similar UV and PL spectra. The electron cloud distributions in their HOMOs of the two compounds were localized on the pyrrolo[3,2- $b]$ pyrrole core and the phenyl groups in the 2,5-positions, while the electron cloud distributions in their LUMOs were primarily distributed on benzene rings in the 1,4-positions. We noted that the electron cloud distributions in HOMOs and LUMOs were spatially separated, implying that there was a charge-transfer process when illuminated by UV. The ICT transition absorption peak at around $370 \mathrm{~nm}$ (Fig. 1) is consistent with the theoretical calculations.

\section{Conclusions}

We synthesized nine TPPP derivatives with different lengths of alkyl chains, and the nine derivatives were all AIE-active. The emission efficiency in the aggregation states exhibited a decreased tendency with increasing the alkyl chain length. TPPP-C6,7,8,12 with longer alkyl chains possessed LC characteristics. The four AIE-active LCs were monotropic liquid crystals, which were formed during the cooling process and assembled into a type of very stabilized LC phase. They could be kept for several months at room temperature and emitted blue-green fluorescence. To the best of our knowledge, this kind of stabilized LC phase with AIE features has not been reported before, and could have significant applications, such as in polarized light emission. Our experimental results showed that the twisted TPPP structure and the planar structure of the pyrrolo[3,2- $b]$ pyrrole core work together to construct AIE-active LCs.

\section{Conflicts of interest}

There are no conflicts to declare.

\section{Acknowledgements}

We are grateful for the support from the National Natural Scientific Foundation of China (Grants 21490574, 51673024, and 21474009) and the National Basic Research Program of China (973 Program; Grant 2013CB834704). W. C. gratefully acknowledges the financial support from the U.S. Department of Energy, Office of Science, Materials Sciences and Engineering Division. We also thank Dr Joseph Strzalka and Dr Zhang Jiang for the assistance with GIWAXS measurements. Use of the Advanced Photon Source (APS) at the Argonne National Laboratory was supported by the U.S. Department of Energy, Office of Science, Office of Basic Energy Sciences, under contract no. DE-AC0206CH11357.

\section{Notes and references}

1 T. Kato, J. Uchida, T. Ichikawa and T. Sakamoto, Angew. Chem., Int. Ed., 2018, 57, 4355-4371.

2 T. Wöhrle, I. Wurzbach, J. Kirres, A. Kostidou, N. Kapernaum, J. Litterscheidt, J. C. Haenle, P. Staffeld, A. Baro, F. Giesselmann and S. Laschat, Chem. Rev., 2016, 116, 1139-1241.

3 H. K. Bisoyi and Q. Li, Acc. Chem. Res., 2014, 47, 3184-3195.

4 L. Wang, H. Dong, Y. Li, R. Liu, Y. F. Wang, H. K. Bisoyi, L. D. Sun, C. H. Yan and Q. Li, Adv. Mater., 2015, 27, 2065-2069.

5 D. Zhao, F. Fan, J. Cheng, Y. Zhang, K. S. Wong, V. G. Chigrinov, H. S. Kwok, L. Guo and B. Z. Tang, Adv. Opt. Mater., 2015, 3, 199-202.

6 D. Zhao, H. He, X. Gu, L. Guo, K. S. Wong, J. W. Y. Lam and B. Z. Tang, Adv. Opt. Mater., 2016, 4, 534-539.

7 B. A. S. Jose, J. Yan and K. Akagi, Angew. Chem., Int. Ed., 2015, 126, 10817-10820.

8 L. Wang, H. Dong, Y. Li, C. Xue, L. D. Sun, C. H. Yan and Q. Li, J. Am. Chem. Soc., 2014, 136, 4480-4483.

9 D. Zhao, F. Fan, V. G. Chigrinov, H. S. Kwok and B. Z. Tang, J. Soc. Inf. Disp., 2015, 23, 218-222.

10 Y. Wang, Y. Liao, C. P. Cabry, D. Zhou, G. Xie, Z. Qu, D. W. Bruce and W. Zhu, J. Mater. Chem. C, 2017, 5, 3999-4008.

11 H. T. Bui, J. Kim, H. J. Kim, B. K. Cho and S. Cho, J. Phys. Chem. C, 2016, 120, 26695-26702.

12 A. S. Matharu, S. Jeeva and P. S. Ramanujam, Chem. Soc. Rev., 2007, 36, 1868-1880.

13 J. Sha, H. Lu, M. Zhou, G. Xia, Y. Fang, G. Zhang, L. Qiu, J. Yang and Y. Ding, Org. Electron., 2017, 50, 177-183.

14 S. J. Yoon, J. H. Kim, K. S. Kim, J. W. Chung, B. Heinrich, F. Mathevet, P. Kim, B. Donnio, A. J. Attiaset, D. Kim and S. Y. Park, Adv. Funct. Mater., 2012, 22, 61-69.

15 J. A. H. P. Sol, V. Dehm, R. Hecht, F. Würthner, A. P. H. J. Schenning and M. G. Debije, Angew. Chem., Int. Ed., 2018, 57, 1030-1033. 
16 Y. Sagara, C. Weder and N. Tamaoki, Chem. Mater., 2017, 29, 6145-6152.

17 H. K. Bisoyi and Q. Li, Chem. Rev., 2016, 116, 15089-15166.

18 J. Mei, N. L. C. Leung, R. T. K. Kwok, J. W. Y. Lam and B. Z. Tang, Chem. Rev., 2015, 115, 11718-11940.

19 J. Luo, Z. Xie, J. W. Y. Lam, L. Cheng, H. Chen, C. Qiu, H. S. Kwok, X. Zhan, Y. Liu, D. Zhu and B. Z. Tang, Chem. Commun., 2001, 1740-1741.

20 Y. Hong, J. W. Y. Lam and B. Z. Tang, Chem. Soc. Rev., 2011, 40, 5361-5388.

21 Q. Li and Z. Li, Adv. Sci., 2017, 4, 1600484.

22 J. Yang, L. Li, Y. Yu, Z. Ren, Q. Peng, S. Ye, Q. Li and Z. Li, Mater. Chem. Front., 2017, 1, 91-99.

23 C. Wang and Z. Li, Mater. Chem. Front., 2017, 1, 2174-2194.

24 X. Zhan, J. Zhang, Y. Gong, S. Tang, J. Tu, Y. Xie, Q. Peng, G. Yu and Z. Li, Mater. Chem. Front., 2017, 1, 2341-2348.

25 J. H. Wan, L. Y. Mao, Y. B. Li, Z. F. Li, H. Y. Qiu, C. Wang and G. Q. Lai, Soft Matter, 2010, 6, 3195-3201.

26 W. Z. Yuan, Z. Q. Yu, P. Lu, C. Deng, J. W. Y. Lam, Z. Wang, E. Q. Chen, Y. Ma and B. Z. Tang, J. Mater. Chem., 2012, 22, 3323-3326.

27 J. Kim, S. Cho and B. K. Cho, Chem. - Eur. J., 2014, 20, 12734-12739.

28 H. Jing, L. Lu, Y. Feng, J. F. Zheng, L. Deng, E. Q. Chen and X. K. Ren, J. Phys. Chem. C, 2016, 120, 27577-27586.

29 W. Z. Yuan, Z. Q. Yu, Y. Tang, J. W. Y. Lam, N. Xie, P. Lu, E. Q. Chen and B. Z. Tang, Macromolecules, 2011, 44, 9618-9628.

30 Y. Guo, D. Shi, Z. W. Luo, J. R. Xu, M. L. Li, L. H. Yang, Z. Q. Yu, E. Q. Chen and H. L. Xie, Macromolecules, 2017, 50, 9607-9616.

31 L. Liu, M. Wang, L. X. Guo, Y. Sun, X. Q. Zhang, B. P. Lin and H. Yang, Macromolecules, 2018, 51, 4516-4524.

32 M. Zhu, Y. Zhuo, K. Cai, H. Guo and F. Yang, Dyes Pigm., 2017, 147, 343-349.

33 J. W. Park, S. Nagano, S. J. Yoon, T. Dohi, J. Seo, T. Seki and S. Y. Park, Adv. Mater., 2014, 26, 1354-1359.
34 H. Lu, L. Qiu, G. Zhang, A. Ding, W. Xu, G. Zhang, X. Wang, L. Kong, Y. Tian and J. Yang, J. Mater. Chem. C, 2014, 2, 1386-1389.

35 Y. Ren, R. Zhang, C. Yan, T. Wang, H. Cheng and X. Cheng, Tetrahedron, 2017, 73, 5253-5259.

36 Y. Ji, Z. Peng, B. Tong, J. Shi, J. Zhi and Y. Dong, Dyes Pigm., 2017, 139, 664-671.

37 Z. Peng, Y. Ji, Z. Huang, B. Tong, J. Shi and Y. Dong, Mater. Chem. Front., 2018, 2, 1175-1183.

38 M. Krzeszewski, B. Thorsted, J. Brewer and D. T. Gryko, J. Org. Chem., 2014, 79, 3119-3128.

39 M. Krzeszewski, D. Gryko and D. T. Gryko, Acc. Chem. Res., 2017, 50, 2334-2345.

40 H. Lu, Y. Zheng, X. Zhao, L. Wang, S. Ma, X. Han, B. Xu, W. Tian and H. Gao, Angew. Chem., Int. Ed., 2016, 55, 155-159.

41 H. Qian, M. E. Cousins, E. H. Horak, A. Wakefield, M. D. Liptak and I. Aprahamian, Nat. Chem., 2017, 9, 83-87.

42 W. Qin, K. Li, G. Feng, M. Li, Z. Yang, B. Liu and B. Z. Tang, Adv. Funct. Mater., 2014, 24, 635-643.

43 J. Mei, Y. Hong, J. W. Y. Lam, A. Qin, Y. Tang and B. Z. Tang, Adv. Mater., 2014, 26, 5429-5479.

44 X. Y. Shen, Y. J. Wang, H. Zhang, A. Qin, J. Z. Sun and B. Z. Tang, Chem. Commun., 2014, 50, 8747-8750.

45 L. Li, M. Salamończyk, S. Shadpour, C. Zhu, A. Jákli and T. Hegmann, Nat. Commun., 2018, 9, 714-721.

46 M. Alaasar, M. Prehm, M. Brautzsch and C. Tschierske, J. Mater. Chem. C, 2014, 2, 5487-5501.

47 W. Chen, T. Xu, F. He, W. Wang, C. Wang, J. Strzalka, Y. Liu, J. Wen, D. J. Miller, J. Chen, K. Hong, L. Yu and S. B. Darling, Nano Lett., 2011, 11, 3707-3713.

48 M. Shao, J. Keum, J. Chen, Y. He, W. Chen, J. F. Browning, J. Jakowski, B. G. Sumpter, I. N. Ivanov, Y. Ma, C. M. Rouleau, S. C. Smith, D. B. Geohegan, K. Hong and K. Xiao, Nat. Commun., 2014, 5, 3180-3181.

49 Y. Yang, W. Chen, L. Dou, W. Chang, H. Duan, B. Bob, G. Li and Y. Yang, Nat. Photonics, 2015, 9, 190-198.

50 L. Lu, T. Xu, W. Chen, E. S. Landry and L. Yu, Nat. Photonics, 2014, 8, 716-722. 